\title{
Reaction Pathways Leading to Arylene Ethynylene Macrocycles via Alkyne Metathesis
}

\author{
Wei Zhang and Jeffrey S. Moore* \\ Roger Adams Laboratory, Departments of Chemistry and Materials Science \& Engineering, University of \\ Illinois at Urbana-Champaign, Urbana, IL 61801,USA.E-mail: jsmoore@uiuc.edu
}

Table 1. Calculated and observed mass of intermediate oligomers shown in Figure 4.

\begin{tabular}{ccc}
\hline product & calcd mass & observed mass \\
\hline linear 18mer $+\mathrm{Na}^{+}$ & 5783 & 5784 \\
cyclic 20mer $+\mathrm{Na}^{+}$ & 5825 & 5825 \\
linear 19mer $+\mathrm{Na}^{+}$ & 6074 & 6074 \\
cyclic 21mer $+\mathrm{Na}^{+}$ & 6115 & 6115 \\
linear 20mer $+\mathrm{Na}^{+}$ & 6364 & 6364 \\
cyclic 22mer $+\mathrm{Na}^{+}$ & 6405 & 6406 \\
\hline
\end{tabular}

Table 2. Calculated and observed mass of cyclic species shown in Figure 7.

\begin{tabular}{lcc}
\hline Compound & calcd mass & observed mass \\
\hline $6 t$-Bu & 936 & 936 \\
$5 t-\mathrm{Bu}+1 \mathrm{Tg}+\mathrm{Na}^{+}$ & 1093 & 1093 \\
$4 t-\mathrm{Bu}+2 \mathrm{Tg}+\mathrm{Na}^{+}$ & 1227 & 1226 \\
$3 t-\mathrm{Bu}+3 \mathrm{Tg}+\mathrm{Na}^{+}$ & 1361 & 1360 \\
$2 t-\mathrm{Bu}+4 \mathrm{Tg}+\mathrm{Na}^{+}$ & 1495 & 1495 \\
$1 t-\mathrm{Bu}+5 \mathrm{Tg}+\mathrm{Na}^{+}$ & 1630 & 1629 \\
$6 \mathrm{Tg}+\mathrm{Na}^{+}$ & 1763 & 1763 \\
\hline
\end{tabular}




\section{Calculation of entropy and enthalpy values of cyclic oligomers: ${ }^{1,2}$}

$$
\begin{aligned}
& \mathrm{ISD}^{3}=10^{27} /[\mathrm{x}] \cdot N_{\mathrm{A}}=10^{27} / 10.35 N_{\mathrm{A}}=160.44 \AA^{3} \\
& \mathrm{ISD}=5.43 \AA \\
& \mathrm{E}_{\text {molec }}=5.02 \AA \\
& \mathrm{V}_{\text {free }}=8\left(\mathrm{ISD}-\mathrm{E}_{\text {molec }}\right)^{3}=8(5.43-5.02)^{3}=0.55 \AA^{3} \\
& V_{\text {free }}=[\mathrm{x}] \cdot N_{\mathrm{A}} \cdot \mathrm{V}_{\text {free }}=3.43 \times 10^{24} \AA^{3}=0.00343 \mathrm{~L}
\end{aligned}
$$

ISD - Intermolecular distance

$[\mathrm{x}]$ - Concentration of molecules

$N_{\mathrm{A}}-$ Avogadro constant

$\mathrm{E}_{\text {molec }}$ - Edge length of a molecule (assuming a small cube)

$\mathrm{V}_{\text {free }}$ - Volume of cube defines motion of center of mass of a single molecule

$V_{\text {free }}$ - Volume of cubes define motions of centers of masses of one mole of molecules

$$
\begin{aligned}
* * * * * * * * * * * * * * * * * * * * * * * * * * * * *) & \\
S_{4 \text { trans }} & =11.1+12.5 \ln (T)+12.5 \ln (M)+8.3 \ln V_{\text {free }} \\
& =11.1+12.5 \ln 303+12.5 \ln 632+8.3 \ln 0.00343 \\
& =11.1+71.42+80.61-47.10 \\
& =116.03 \mathrm{~J} / \mathrm{mol} \cdot \mathrm{K}
\end{aligned}
$$




$$
\begin{aligned}
& =25.0 \ln 790+12.5 \ln 303-74.1 \\
& =166.80+71.42-74.1 \\
& =164.12 \mathrm{~J} / \mathrm{mol} \cdot \mathrm{K}
\end{aligned}
$$

$$
H_{5 \mathrm{f}}=-26.752 \mathrm{kcal} / \mathrm{mol}=-111.82 \mathrm{~kJ} / \mathrm{mol}
$$

$$
\begin{aligned}
S_{6 \text { trans }} & =11.1+12.5 \ln (T)+12.5 \ln (M)+8.3 \ln V_{\text {free }} \\
& =11.1+12.5 \ln 303+12.5 \ln 948+8.3 \ln 0.00343 \\
& =11.1+71.42+85.68-47.10 \\
& =121.1 \mathrm{~J} / \mathrm{mol} \cdot \mathrm{K}
\end{aligned}
$$

(Hexacycle translational entropy)

$$
\begin{aligned}
S_{6 \text { rot }} & =25.0 \ln (M)+12.5 \ln (T)-74.1 \\
& =25.0 \ln 948+12.5 \ln 303-74.1 \\
& =171.36+71.42-74.1 \\
& =168.68 \mathrm{~J} / \mathrm{mol} \cdot \mathrm{K}
\end{aligned}
$$

$H_{6 \mathrm{f}}=-36.067 \mathrm{kcal} / \mathrm{mol}=-151.48 \mathrm{~kJ} / \mathrm{mol}$

$$
\begin{aligned}
S_{7 \text { trans }} & =11.1+12.5 \ln (T)+12.5 \ln (M)+8.3 \ln V_{\text {free }} \\
& =11.1+12.5 \ln 303+12.5 \ln 1106+8.3 \ln 0.00343 \\
& =11.1+71.42+87.61-47.10 \\
& =123.03 \mathrm{~J} / \mathrm{mol} \cdot \mathrm{K}
\end{aligned}
$$

$$
\begin{aligned}
S_{7 \mathrm{rot}} & =25.0 \ln (M)+12.5 \ln (T)-74.1 \\
& =25.0 \ln 1106+12.5 \ln 303-74.1 \\
& =175.21+71.42-74.1 \\
& =172.53 \mathrm{~J} / \mathrm{mol} \cdot \mathrm{K}
\end{aligned}
$$

$$
H_{7 \mathrm{f}}=-41.228 \mathrm{kcal} / \mathrm{mol}=-172.33 \mathrm{~kJ} / \mathrm{mol}
$$

$$
\begin{aligned}
S_{\text {8trans }} & =11.1+12.5 \ln (T)+12.5 \ln (M)+8.3 \ln V_{\text {free }} \\
& =11.1+12.5 \ln 303+12.5 \ln 1264+8.3 \ln 0.00343 \\
& =11.1+71.42+89.28-47.10 \\
& =124.70 \mathrm{~J} / \mathrm{mol} \cdot \mathrm{K} \quad \quad \text { (Octacycle translational entropy) }
\end{aligned}
$$

$$
\begin{aligned}
S_{8 \text { rot }} & =25.0 \ln (M)+12.5 \ln (T)-74.1 \\
& =25.0 \ln 1264+12.5 \ln 303-74.1 \\
& =178.55+71.42-74.1 \\
& =175.87 \mathrm{~J} / \mathrm{mol} \cdot \mathrm{K}
\end{aligned}
$$

$$
H_{8 \mathrm{f}}=-46.779 \mathrm{kcal} / \mathrm{mol}=-195.54 \mathrm{~kJ} / \mathrm{mol}
$$




$$
\begin{aligned}
\Delta G_{6 \rightarrow 4} & =\Delta H-\mathrm{T} \Delta S \\
& =\left(6 H_{4 \mathrm{f}}-4 H_{6 \mathrm{f}}\right)-\mathrm{T}\left(6 S_{4 \text { trans }}+6 S_{4 \mathrm{rot}}-4 S_{6 \text { trans }}-4 S_{6 \mathrm{rot}}\right) \\
& =(-6 \times 32.90+4 \times 151.48)-303(6 \times 0.116+6 \times 0.159-4 \times 0.121-4 \times 0.169) \\
& =408.52-303 \times 0.490 \\
& =260.05 \mathrm{~kJ} / \mathrm{mol} \\
& =62.21 \mathrm{kcal} / \mathrm{mol}
\end{aligned}
$$

$\Delta G_{6 \rightarrow 4}=15.6 \mathrm{kcal} / \mathrm{mol}$ (per mole hexacycle)

\section{5 hexacycle $\rightleftharpoons 6$ pentacycle}

$$
\begin{aligned}
\Delta G_{6 \rightarrow 5} & =\Delta H-\mathrm{T} \Delta S \\
& =\left(6 H_{5 \mathrm{f}}-5 H_{6 \mathrm{f}}\right)-\mathrm{T}\left(6 S_{5 \text { trans }}+6 S_{5 \mathrm{rot}}-5 S_{6 \text { trans }}-5 S_{6 \mathrm{rot}}\right) \\
& =(-6 \times 111.82+5 \times 151.48)-303(6 \times 0.119+6 \times 0.164-5 \times 0.121-5 \times 0.169) \\
& =86.48-303 \times 0.248 \\
& =11.34 \mathrm{~kJ} / \mathrm{mol} \\
& =2.71 \mathrm{kcal} / \mathrm{mol} \\
\Delta G_{6 \rightarrow 5} & =0.54 \mathrm{kcal} / \mathrm{mol} \text { (per mole hexacycle) }
\end{aligned}
$$

7 hexacycle $\rightleftharpoons 6$ heptacycle

$$
\begin{aligned}
& \Delta G_{6 \rightarrow 7}=\Delta H-\mathrm{T} \Delta S \\
& =\left(6 H_{7 \mathrm{f}}-7 H_{6 \mathrm{f}}\right)-\mathrm{T}\left(6 S_{7 \text { trans }}+6 S_{7 \mathrm{rot}}-7 S_{6 \text { trans }}-7 S_{6 \mathrm{rot}}\right) \\
& =(-6 \times 172.33+7 \times 151.48)-303(6 \times 0.123+6 \times 0.173-7 \times 0.121-7 \times 0.169) \\
& =26.38-303 \times(-0.254) \\
& =103.34 \mathrm{~kJ} / \mathrm{mol} \\
& =24.72 \mathrm{kcal} / \mathrm{mol}
\end{aligned}
$$

\section{8 hexacycle $\rightleftharpoons 6$ octacycle}

$$
\begin{aligned}
\Delta G_{6 \rightarrow 8} & =\Delta H-\mathrm{T} \Delta S \\
& =\left(6 H_{8 \mathrm{f}}-8 H_{6 \mathrm{f}}\right)-\mathrm{T}\left(6 S_{8 \text { trans }}+6 S_{8 \mathrm{rot}}-8 S_{6 \text { trans }}-8 S_{6 \mathrm{rot}}\right) \\
& =(-6 \times 195.54+8 \times 151.48)-303(6 \times 0.125+6 \times 0.176-8 \times 0.121-8 \times 0.169) \\
& =38.60-303 \times(-0.514) \\
& =194.34 \mathrm{~kJ} / \mathrm{mol} \\
& =46.49 \mathrm{kcal} / \mathrm{mol} \\
\Delta G_{6 \rightarrow 8} & =5.81 \mathrm{kcal} / \mathrm{mol} \text { (per mole hexacycle) }
\end{aligned}
$$


5 hexacycle $\rightleftharpoons 6$ pentacycle

$\Delta G_{6 \rightarrow 5}=\Delta H-\mathrm{T} \Delta S=-\mathrm{RT} \ln \mathrm{K}$

$-\operatorname{lnK}=(\Delta H / \mathrm{R}) \cdot 1 / \mathrm{T}-\Delta S / \mathrm{R}$

$\Delta H$ and $\Delta S$ value can be calculated using the above equation

I. $95{ }^{\circ} \mathrm{C}$

$[$ hexacycle $]=0.0363 \times(2.85 \times 6) /(2.85 \times 6+1.0 \times 5) \times 6=0.00468$

$[$ pentacycle $]=0.0363 \times(1.0 \times 5) /(2.85 \times 6+1.0 \times 5) \times 5=0.00164$

$-\ln \mathrm{K}_{1}=-\ln [\text { pentacycle }]^{6} /[\text { hexacycle }]^{5}=11.67$

$1 / \mathrm{T}_{1}=1 / 368=0.00272$

II. $75^{\circ} \mathrm{C}$

$[$ hexacycle $]=0.0363 \times(3.40 \times 6) /(3.40 \times 6+1.0 \times 5) \times 6=0.00486$

$[$ pentacycle $]=0.0363 \times(1.0 \times 5) /(3.40 \times 6+1.0 \times 5) \times 5=0.00143$

$-\ln K_{2}=-\ln [\text { pentacycle }]^{6} /[\text { hexacycle }]^{5}=12.67$

$1 / \mathrm{T}_{2}=1 / 348=0.00287$

III. $60{ }^{\circ} \mathrm{C}$

$[$ hexacycle $]=0.0363 \times(4.17 \times 6) /(4.17 \times 6+1.0 \times 5) \times 6=0.00504$

$[$ pentacycle $]=0.0363 \times(1.0 \times 5) /(4.17 \times 6+1.0 \times 5) \times 5=0.00121$

$-\ln K_{3}=-\ln [\text { pentacycle }]^{6} /[\text { hexacycle }]^{5}=13.85$

$1 / \mathrm{T}_{3}=1 / 333=0.00300$

IV. $45^{\circ} \mathrm{C}$

$[$ hexacycle $]=0.0363 \times(5.13 \times 6) /(5.13 \times 6+1.0 \times 5) \times 6=0.00520$

$[$ pentacycle $]=0.0363 \times(1.0 \times 5) /(5.13 \times 6+1.0 \times 5) \times 5=0.00101$ 
$-\ln \mathrm{K}_{4}=-\ln [\text { pentacycle }]^{6} /[\text { hexacycle }]^{5}=15.09$

$1 / \mathrm{T}_{4}=1 / 318=0.00314$

V. $30^{\circ} \mathrm{C}$

$[$ hexacycle $]=0.0363 \times(6.51 \times 6) /(6.51 \times 6+1.0 \times 5) \times 6=0.00536$

$[$ pentacycle $]=0.0363 \times(1.0 \times 5) /(6.51 \times 6+1.0 \times 5) \times 5=0.000824$

$-\ln K_{5}=-\ln [\text { pentacycle }]^{6} /[\text { hexacycle }]^{5}=16.46$

$1 / \mathrm{T}_{5}=1 / 303=0.00330$

Plot $-\operatorname{lnK}$ vs. $1 / \mathrm{T}$ as follows:

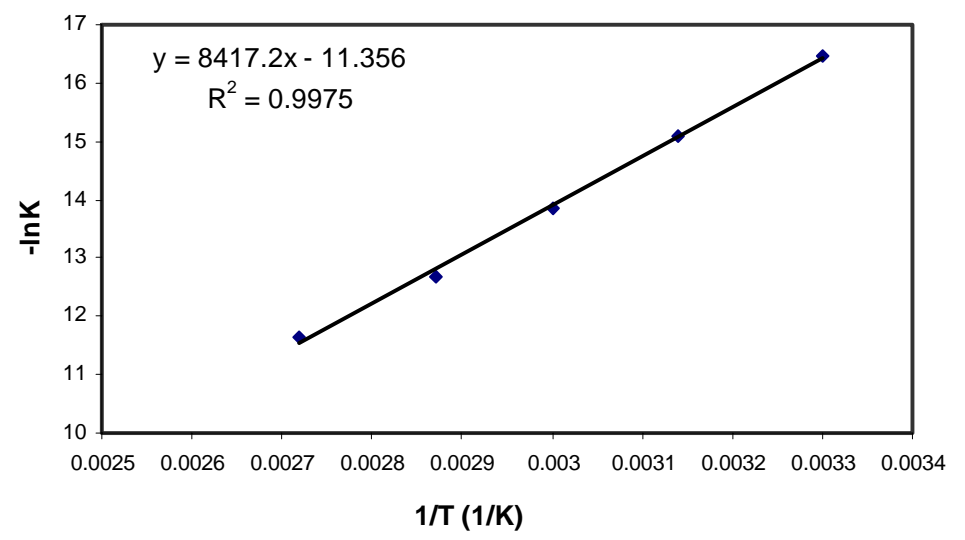

$\Delta H / \mathrm{R}=8417.2$

$\Delta H=8.31 \times 8417.2 \mathrm{~J} / \mathrm{mol}=16.75 \mathrm{kcal} / \mathrm{mol}$

$\Delta H=3.4 \mathrm{kcal} / \mathrm{mol}$ (per mole hexacycle, theoretical calculation is $4.1 \mathrm{kcal} / \mathrm{mol})^{2}$

$\Delta S / \mathrm{R}=11.356$

$\Delta S=8.31 \times 11.356 \mathrm{~J} / \mathrm{mol} \cdot \mathrm{K}=0.0226 \mathrm{kcal} / \mathrm{mol} \cdot \mathrm{K}$

$\Delta S=0.005 \mathrm{kcal} / \mathrm{mol} \cdot \mathrm{K}(\text { per mole hexacycle, theoretical calculation is } 0.012 \mathrm{kcal} / \mathrm{mol} \cdot \mathrm{K})^{1}$ 
Diarylacetylene byproduct characterization:

${ }^{1} \mathrm{H}$ NMR $\left(\mathrm{CDCl}_{3}, 500 \mathrm{MHz}\right): \delta 7.92(\mathrm{~d}, J=8.1 \mathrm{~Hz}, 4 \mathrm{H}), 7.85(\mathrm{~m}, 4 \mathrm{H}), 7.75(\mathrm{~d}, J=8.1$ $\mathrm{Hz}, 4 \mathrm{H}), 7.68$ (br s, 8H), $7.62(\mathrm{~m}, 2 \mathrm{H}), 7.52(\mathrm{~m}, 4 \mathrm{H})$; EI-MS $\left(\mathrm{C}_{40} \mathrm{H}_{26} \mathrm{O}_{2}\right.$, calcd 538.2): m/z (\%): $\mathrm{M}^{+} 538.2(53), 371.2(25), 220.1(61), 91.1(100)$.

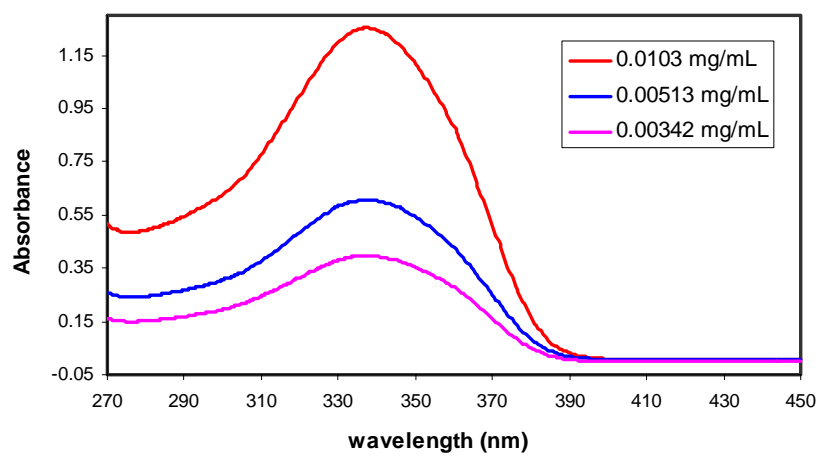

Figure 1. UV spectra of diarylacetylene byproduct in $\mathrm{CHCl}_{3}$ at different concentrations $\left(25^{\circ} \mathrm{C}\right)$.

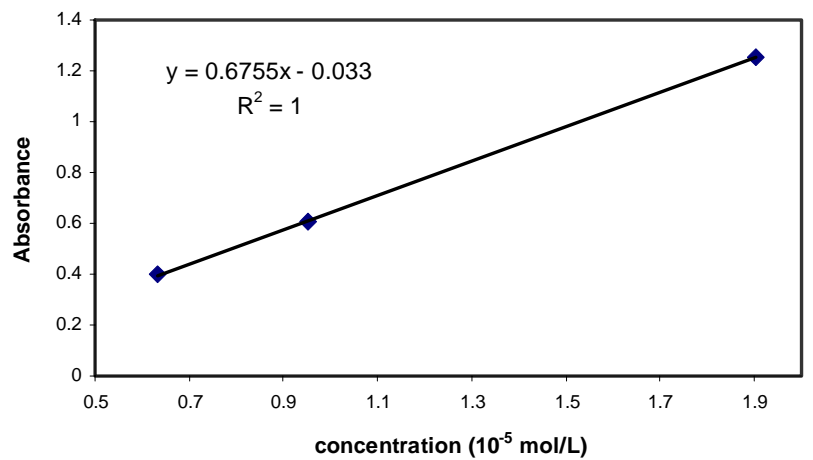

Figure 2. Absorbance changes of diarylacetylene byproduct at different concentrations in $\mathrm{CHCl}_{3}\left(25^{\circ} \mathrm{C}\right)$.

Based on Lambert-Beer law: $A=\varepsilon b c$

$\varepsilon=6.8 \times 10^{4} \mathrm{~L} / \mathrm{mol} \cdot \mathrm{cm}$ 


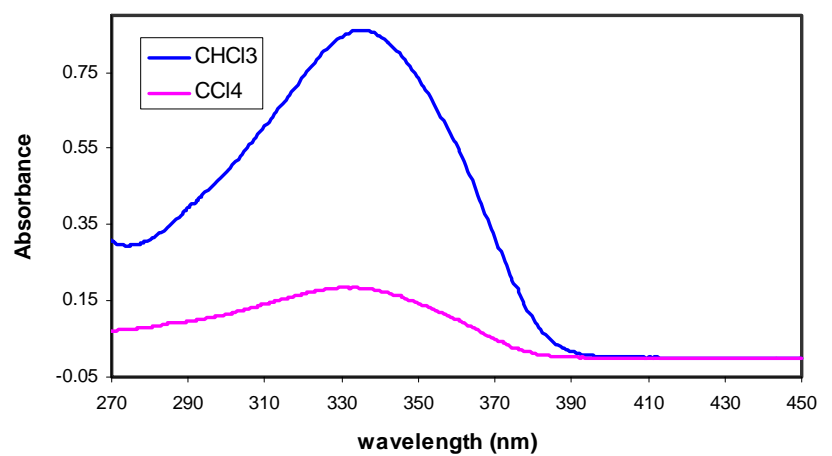

Figure 3. UV spectra of diarylacetylene byproduct in different solvents. The spectra were recorded at $1 / 4$ of the saturated concentrations of the byproduct at $25{ }^{\circ} \mathrm{C}$. In $\mathrm{CHCl}_{3}$, the spectrum was recorded using the cell with $1 \mathrm{~cm}$ length. In $\mathrm{CCl}_{4}$, the spectrum was recorded using the cell with $10 \mathrm{~cm}$ length.

The saturated concentration of diarylacetylene byproduct in $\mathrm{CHCl}_{3}$ can be calculated:

$c=4 \times 0.861 / 6.8 \times 10^{4}=5.1 \times 10^{-5} \mathrm{~mol} / \mathrm{L}$

The saturated concentration of diarylacetylene byproduct in $\mathrm{CCl}_{4}$ can be calculated:

$c=4 \times 0.185 / 10 \times 6.8 \times 10^{4}=1.1 \times 10^{-6} \mathrm{~mol} / \mathrm{L}$

\section{References:}

1. The entropy values of methyl benzoate macrocycles are estimated based on a semiquantitative model, see Mammen, M.; Shakhnovich, E. I.; Deutch, J. M.; Whitesides, G. M. J. Org. Chem. 1998, 63, 3821-3830.

2. Spartan software (version 4.0, Wavefunction, Inc., Irvine, California) was used to estimate the enthalpy values of methyl benzoate macrocycles in AM1 level. 